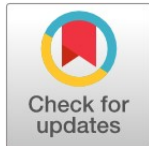

PRIMARY RESEARCH

\title{
Defect formation in EBM parts built at different orienta- tion
}

\author{
C. Camilleri ${ }^{1 *}$, A. Rochman ${ }^{2}$ \\ ${ }^{1,2}$ Department of Industrial and Manufacturing Engineering, University of Msida, Msida, Malta
}

\author{
Keywords \\ Additive manufacturing \\ EBM orientation \\ Dimensional analysis \\ Ti-6Al-4V
}

Received: 4 April 2019

Accepted: 7 May 2019

Published: 28 June 2019

\begin{abstract}
This study presents the results of an analysis carried to compare the dimensional quality of parts built at different orientations and identify causes of dimensional inaccuracies of electron beam melting (EBM) parts built in different scenarios. The scenarios considered reflect common overhanging positions of part features built in the EBM machine. The results show that the parts built on the support structure had the optimum dimensional accuracy. Furthermore, there are close quality characteristics of the cylindrical features built in the $\mathrm{X}$ and $\mathrm{Y}$ direction, i.e., perpendicular to the build direction, due to the alternating scanning direction adopted by the EBM machine.
\end{abstract}

(C) 2019 The Author(s). Published by TAF Publishing.

\section{INTRODUCTION}

The EBM embodies the latest, state-of-the-art technology in the Additive Manufacturing industry. Despite being relatively new on the market, it is already widely recognized as very valuable for the next generation of advanced manufacturing. Its suitability for the fabrication of customized products, such as customized hip stem prostheses in the medical sector, the superior material properties combined with the relatively low lead time, etc., gives the EBM technology a significant competitive edge in the advanced manufacturing industry $[1,2]$.

However, this technology also has its drawbacks, namely the rough surface finish produced and its limited control on dimensional accuracy, particularly due to the "Stair Casing Effect" [3]. This has led to the widespread impression that the EBM machine often produces near-net-shape parts that require a subsequent processing treatment. Dimensional inaccuracy and high surface roughness also relate to properties, such as the wear rate and friction, which directly affect the lifetime of the product $[4,5,6]$. [7] indicate that it is very challenging to obtain high dimensional accuracy EBM and other AM built parts. This is attributed to various factors including material behavior, building strategy, and orientation.

Part orientation is one of the major parameters that affect the quality of built parts. Part orientation defines the rotation of the built parts in the build space around the axes of the machine's coordinate system $[8,9]$. [10] argue that determining the most suitable orientation is essential to safeguard the part quality as well as lower the manufacturing costs. Moreover, a study by [11] shows that parts built along horizontal orientation are more susceptible to distortions in dimensional accuracy particularly, due to the effect of supports used and the building strategy. Within this context, the aim of this study is to analyse quality issues of EBM built parts vis-a-vis the built orientation. This would provide scholars and EBM part designers with a set of casestudy guidelines which can be used to address these quality issues.

\footnotetext{
${ }^{*}$ Corresponding author: C. Camilleri

†email: christian.camilleri@mcast.edu.mt
} 


\section{A. $E B M$}

The EBM process is primarily subdivided into three major processes, i.e., the pre-fabrication process, the fabrication process, and the post-fabrication process. The prefabrication procedure commences with the development of a solid Computer Aided Design (CAD) model. The file is then converted to STL format, where the 3D CAD model is represented as a collection of triangular facets $[12,13]$. The positioning of the model is an essential part of the EBM process and is critical to the achievement of a good combination of quality and the processing time. The use of a support structure is important when developing a CAD model that has a considerable area with down-facing triangles. The last stage is the slicing process which is done with the EBM Build Assembler TM, where the STL file is cut into horizontal cross-sections that become the tool path that drives the electron beam [14].

The EBM melting process takes place inside a vacuum to eliminate the energy losses from the interactions between electrons and air or gas molecules. The fabrication process initiates by the spreading of a uniform layer of metal powder over the building platform by means of a calibrated rake. A preheat treatment lightly sinters the metal powder, which combines it to ensure stability during the melting process [15].

The electron beam melting process commences with the electron beam gun; this incorporates a tungsten filament that is cooled by a series of water chillers. The filament is heated to extremely high temperatures (exceeding 25000C) by means of a current that is controlled by an electrode. At such high temperature, the filament releases electrons that are accelerated towards the workpiece by means of an electric field. This electric field is created by applying a high voltage of circa $60,000 \mathrm{~V}$ between the filament and the anode [16]. Electromagnetic coils and astigmatism are used to focus the high-velocity electrons over a very small area, typically $0.0025 \mathrm{~mm}$ in diameter [17].

Melting is achieved when the high-velocity electrons strike the powder surface. In this process, the kinetic energy of electrons is rapidly converted to heat which causes the temperature of the metal powder to rapidly exceed its melting temperature, fusing the metal powder into a solidified layer. Initially, the electron beam melts the contours, i.e., the outline perimeter of the respective layer, which is then followed by melting inside the hatch area [18]. Once a layer is completed, the build plate is lowered by one layer thickness, the metal powder is distributed, and the whole process is repeated [19].
After the building process has been completed, the part is cooled down either in a vacuum or by means of a helium flow. A cleaning process is carried out in the recovery cabinet using a combined system of high-pressure air and metal powder to remove the sintered powder.

\section{MATERIALS AND METHOD}

A case study CAD model, composed of three cylindrical parts, with each part oriented in the $X, Y$ and $Z$ direction of the build plate, was developed. As illustrated in Figure 1, the $X$ and $Y$ directions refer to the part features built in horizontal orientation to the start plane. The cylindrical feature built in the $Z$ direction refers to the part feature built in the vertical orientation perpendicular to the build direction and start plane. The diameter of each cylindrical part was specified at $8,000 \mu \mathrm{m}$, whilst the length was specified at $28,000 \mu \mathrm{m}$. The cylinder in the $X$ direction was marked with two rings, whilst the cylinder in the $Y$ direction was marked with one ring.

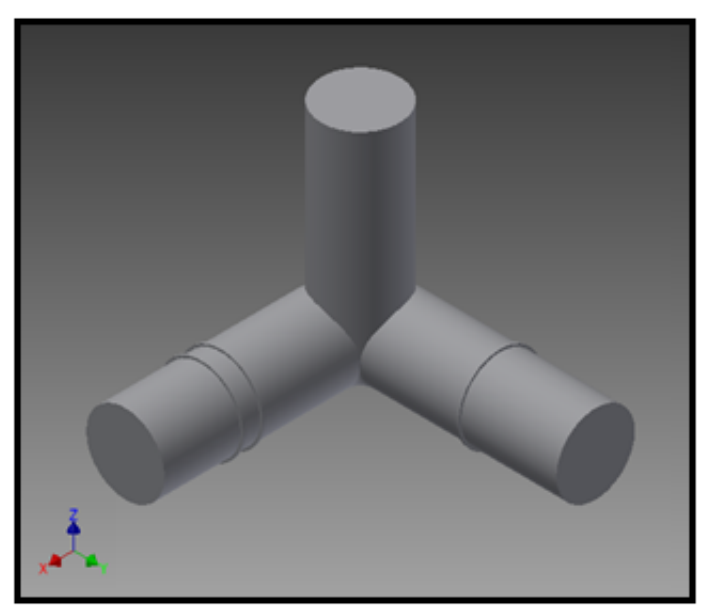

Fig. 1. CAD model of the case study part

The CAD model was inserted in the machine's software and configured to ensure that the $X$ and $Y$ axes were aligned with the orientation of the base plate. The process parameters specified for this experiment are indicated in Table 1. Ti-6Al-4V powder having a mean diameter of $60 \mu \mathrm{m}$ was used to produce the models. The Ti-6Al-4V powder was supplied by Arcam AB (Sweden) and the parts were fabricated in an Arcam S400 EBM machine. After the parts were built, the powder recovery procedure was carried out to remove the powder semi-sintered around the built parts. The CAD model was built in the EBM under four different scenarios. In the first scenario, the part, seen in Figure 2A, was built directly on the base plate so as to ensure an efficient heat transfer from the part to the base plate. The 
second scenario investigated involved a part model built on a support structure with part features in the $X, Y$, and $Z$ directions, as indicated in Figure 2B. The part model was offset from the base plate by $3,000 \mu \mathrm{m}$.

\section{TABLE 1}

EBM PROCESSING PARAMETERS

\begin{tabular}{ll}
\hline \hline Processing Parameter & Value \\
\hline $\begin{array}{l}\text { Preheating Parameter } \\
\text { Beam current }(\mathrm{mA})\end{array}$ & 38 \\
$\begin{array}{l}\text { Focus offset }(\mathrm{mA}) \\
\text { Beam speed }(\mathrm{mm} / \mathrm{s})\end{array}$ & 3 \\
Contour Parameter & 14600 \\
Beam current $(\mathrm{mA})$ & 50 \\
Focus offset outer/inner (mA) & 3 \\
$\begin{array}{l}\text { Beam speed outer/inner }(\mathrm{mm} / \mathrm{s}) \\
\text { Hatch Parameter }\end{array}$ & $340 / 800$ \\
Beam current $(\mathrm{mA})$ & 15 \\
Focus offset outer/inner $(\mathrm{mA})$ & 5 \\
Beam speed outer/inner $(\mathrm{mm} / \mathrm{s})$ & 4530 \\
\hline \hline
\end{tabular}
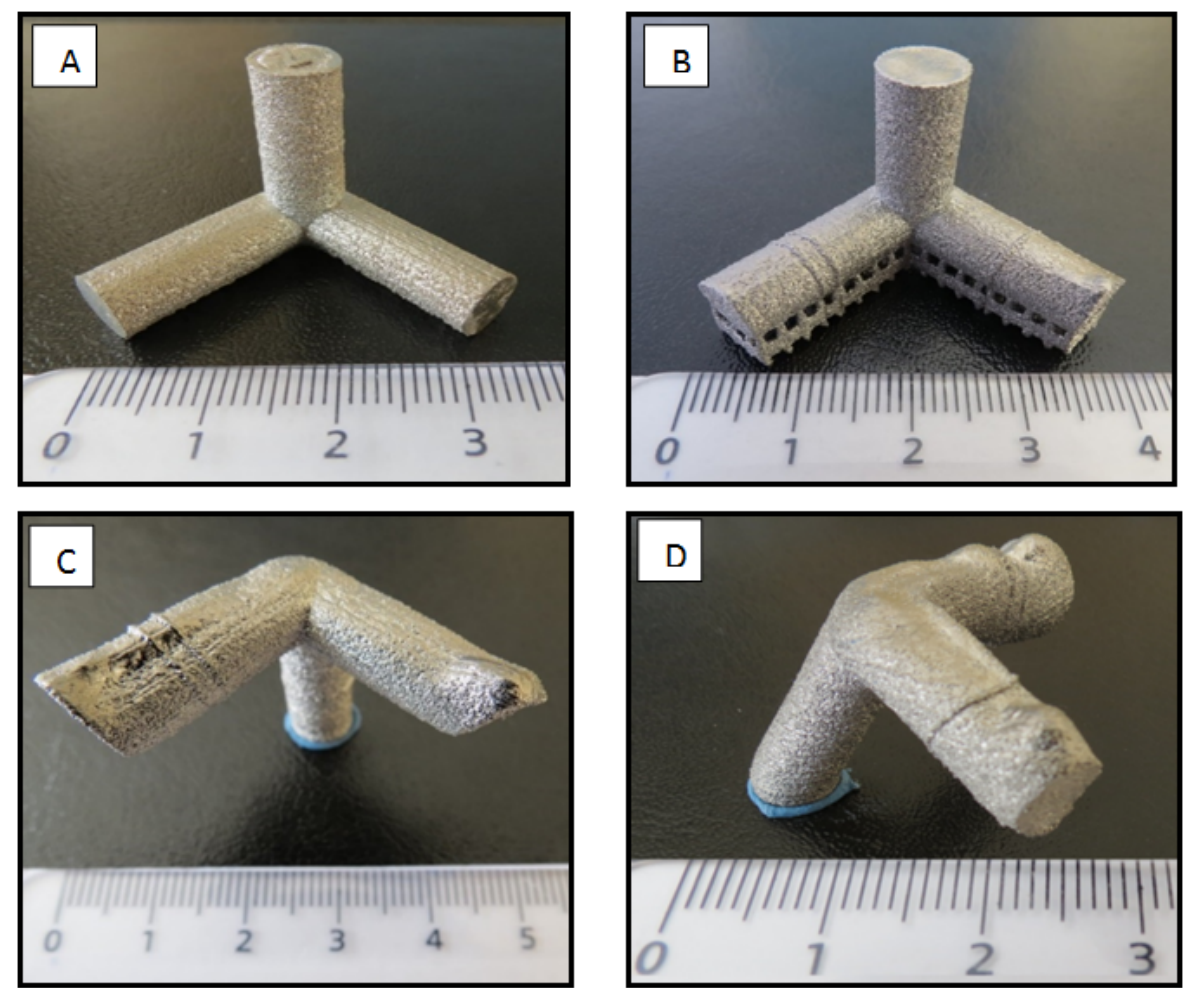

Fig. 2. (A) Scenario 1-Part built in contact with the base plate; (B) Scenario 2-Parts built on the support structure; (C) Scenario 3-Part features in the $X$ and $Y$ directions built on sintered; (D): Scenario 4-Overhanging part features

In order to analyse the dimensional variations with respect to the actual model diameter, each cylindrical feature was cut 3,000 $\mu \mathrm{m}$ away from the surface and the resulting circular cross-sections were analysed using the stereomicroscope. The maximum offset diameter of each circular feature was recorded using a stereomicroscope with a mag-
The purpose of investigating the third scenario was to analyse the deviation from the circularity of features built directly on sintered powder. As indicated in Figure 2C, the circular feature in the $Z$ direction was placed in contact with the base plate, whilst the circular features in the $X$ and $Y$ directions were built directly on sintered powder without the use of support structures. In the fourth scenario, the dimensional analysis of overhanging circular cylinders built at an angle was also investigated. As illustrated in Figure 2D, the circular feature in the $Z$ direction was placed in contact with the base plate at a $60^{\circ}$ angle. The same experimental procedure was repeated and a cross-sectional image of each cylindrical feature was taken using a stereomicroscope. nification of $1000 \mu \mathrm{m}$. For each combination of scenario and orientation position, three readings were taken and the mean values were computed. 


\section{RESULTS AND DISCUSSION}

\section{A. Scenario 1-Part Built in Contact with the Base Plate}

Inspecting the part features under scenario 1, indicated in Figure 3 , it can be noted that the cross-section view of the cylindrical parts in the $X$ and $Y$ directions differs significantly from the cross-section in the $Z$ direction. It can be observed that whilst the upper half of the cross-section view in the $X$ and $Y$ directions resembles the circular feature, the lower half, in contrast, is characterised by three straight edges at its bottom region. The formation of the straight edges, instead of the circular curvature in the $X$ and $Y$ directions, are primarily because the parts were built directly on the base plate.

This indicates that the first few melted layers adhere to the base plate instead of adhering to the part being built. Whilst this effect on part quality can be minimal when large, flat surfaces are being produced, it could become significant when considering small horizontal, circular features. Although the base plate in Figure 3D was not the one used for the build being analyzed, it can clearly be observed that the first few layers of the previous model have adhered to the base plate, thereby creating geometrical irregularities at the surface. This effect not only diminishes the quality of the part being built but also affects the subsequent builds.
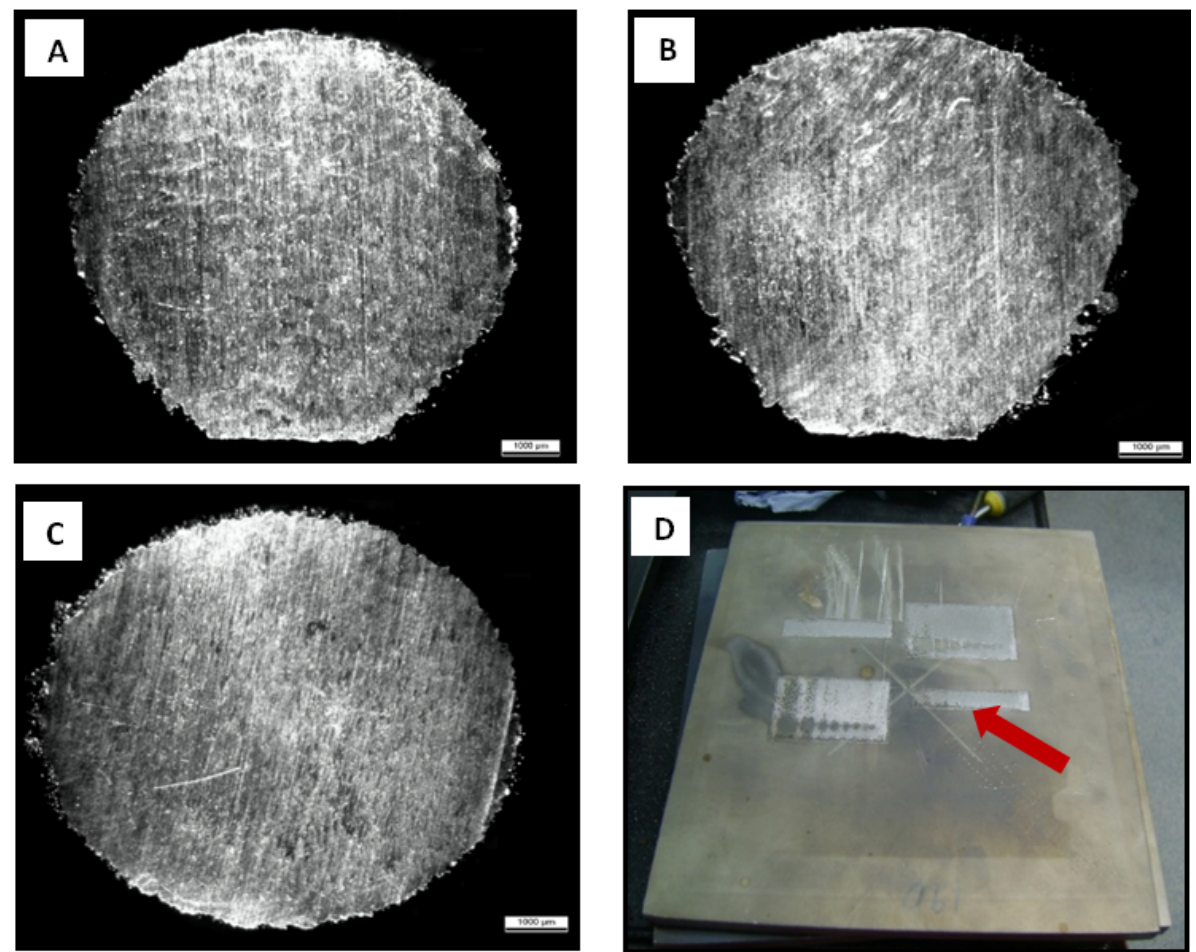

Fig. 3. Part 1 (A)-Cross-sectional view $X$ direction (B)-Cross-sectional view $Y$ direction

(C)-Cross-sectional view $Z$ direction (D)-Base plate with melted layers adhered to it

One-sample $t$-test analysis was carried out to determine whether the sample means obtained for the part features built in $X, Y$, and $Z$ axes are statistically different from the known population mean of $8,000 \mu \mathrm{m}$. In this case, the Null hypothesis specifies that the sample mean is comparable to the specified value and is accepted if the p-value exceeds the 0.05 level of significance. The alternative hypotheses spec- ify that the sample mean differs significantly from the specified value and is accepted if the $p$-value is less than the 0.05 criterion. From the results shown in Table 2, it can be observed that the p-values obtained for the $X, Y$, and $Z$ axes are above the 0.05 level significance, indicating that despite the defect indicated earlier, the measurements obtained are comparable to the sample mean.

TABLE 2

ONE SAMPLE $T$-TEST ANALYSIS FOR SCENARIO 1

\begin{tabular}{llllll}
\hline \hline & $N$ & Mean & Std. Deviation & Std. Error Mean & $p$-Value \\
\hline Diameter $(X$-axis) & 3 & 8230.0700 & 524.03646 & 302.55259 & .526 \\
Diameter $(Y$-axis) & 3 & 7724.7117 & 481.84246 & 278.19188 & .427 \\
Diameter $(Z$-axis $)$ & 3 & 7944.5633 & 139.73804 & 80.67780 & .563 \\
\hline \hline
\end{tabular}




\section{B. Scenario 2-Parts Built on the Support Structure}

Similar to the previous scenario, the cross-sections of the part features built in the $X, Y$, and $Z$ directions were analyzed. In this case, it can also be observed from Figure 4 that the $Z$-axis has better dimensional accuracy when compared to the features built in the $X$ and $Y$ directions. The lat-
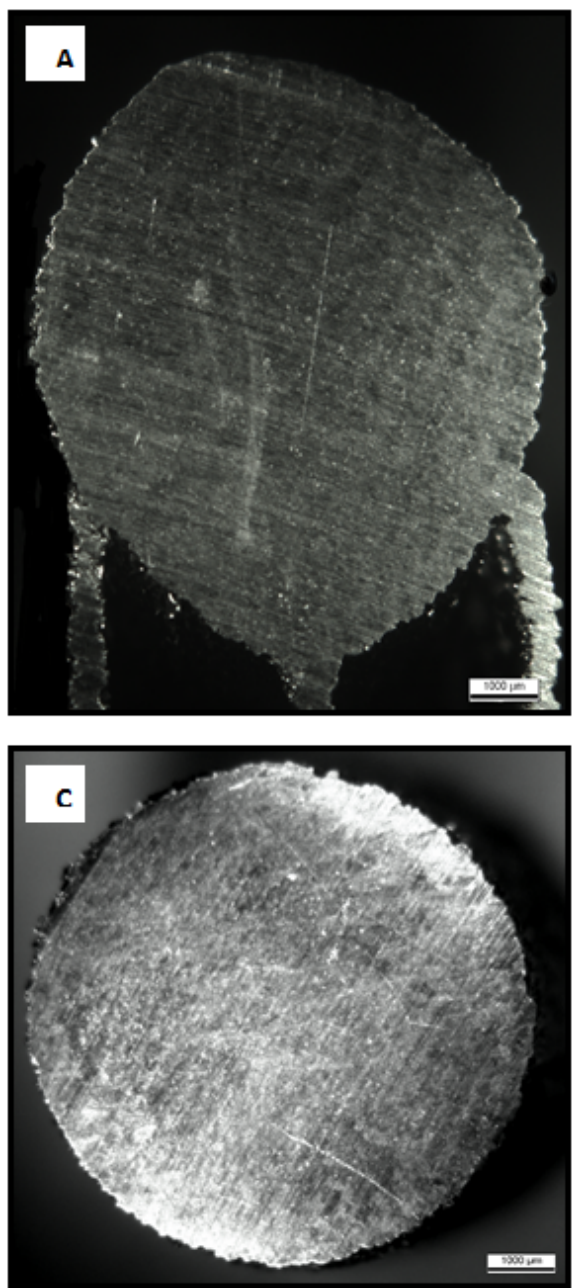

Fig. 4. Part 2 (A)-Cross-sectional view $X$ direction (B)-Cross-sectional view $Y$ direction (C)-Cross-sectional view $Z$ direction

The shrinkage occurs mainly as a result of solidification contraction, i.e., as the cooling progresses, the solidifying layers reduce in size, resulting in increased tension inside the part. Although the support structure of the cylinder was placed in such a way to cover the surface up to almost half the diameter, the tear-drop formation is still significant. This is mainly due to the fact that since the cross-section being investigated has a relatively small diameter, and thus a small layer width, the shrinkage effect will be more significant compared to cross-sections with larger diameters. Thereby, the tear-drop formation can be minimised by increasing the ter parts are characterized by the 'tear-drop formation' resulting in a visible deviation from circularity. This indicates that the tear-drop formation is caused by the shrinkage of the material toward the part's centre during the part's cooling. This 'tear-drop' formation is also reported in studies carried out on parts built via Selective Laser Melting (SLM) [11].

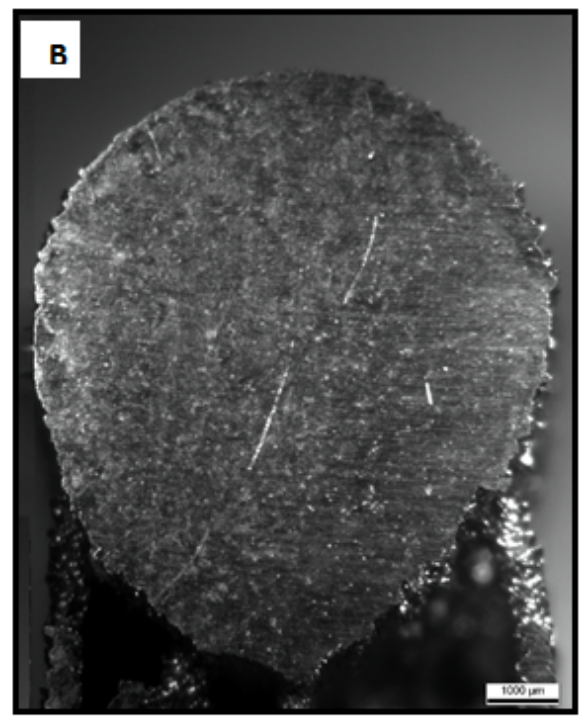

radius of the curvature, when possible, or altering the part orientation or increasing the support structures.

One-sample $t$-test analysis was carried out to the sample mean with the specified diameter. Based on the same hypothesis adopted in the first scenario, from the results in Table 3, it can be observed that the $p$-values obtained for the $X, Y$, and $Z$ axes exceed the 0.05 criterion, indicating that the measurements obtained are comparable to the sample mean. 
TABLE 3

ONE SAMPLE $T$-TEST ANALYSIS FOR SCENARIO 2

\begin{tabular}{llllll}
\hline \hline & $N$ & Mean & Std. Deviation & Std. Error Mean & $p$-Value \\
\hline Diameter $(X$-axis $)$ & 3 & 8015.7567 & 145.65975 & 84.09670 & .187 \\
Diameter $(Y$-axis $)$ & 3 & 7864.9433 & 253.68707 & 146.46630 & 0.454 \\
Diameter $(Z$-axis) & 3 & 7752.1733 & 168.39870 & 39.73295 & .126 \\
\hline \hline
\end{tabular}

\section{Scenario 3-Overhanging Parts}

In the third scenario, as can be seen in Figure 5, in terms of dimensional accuracy, the same pattern observed in the previous scenario can be observed in the scenario as well, i.e., whilst there is minimal dimensional variation in the $Z$
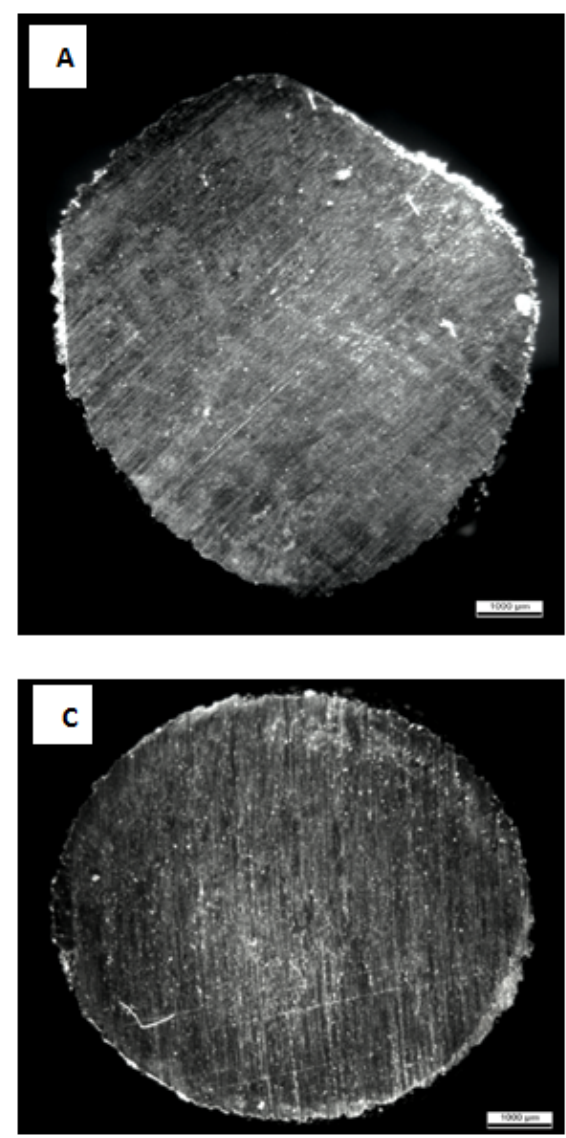

Fig. 5. Part 3 (A)-Cross-sectional view $X$ direction (B)-Cross-sectional view $Y$ direction (C)-Cross-sectional view $Z$ direction, (D) Schematic diagram of part indicating the heat transfer direction

Besides the tear-drop structure, another defect that emerged clearly in this scenario is the swelling produced on the circular features in the $X$ and $Y$ directions. The swelling present on the two circular cylinders in the $X$ and $Y$ directions indicates that the heat retained in the part during and just after melting is larger than the energy generated in the parts produced in the previous scenario. As can be observed in Figure 5D, this can be attributed to the ineffective direction, more severe dimensional inaccuracy can be observed in the $X$ and $Y$ directions. A larger tear-drop formation observed in this scenario can be attributed to the lack of a support structure. Since support structures were not used, the cooling process was prolonged, causing shrinkage to be more severe.
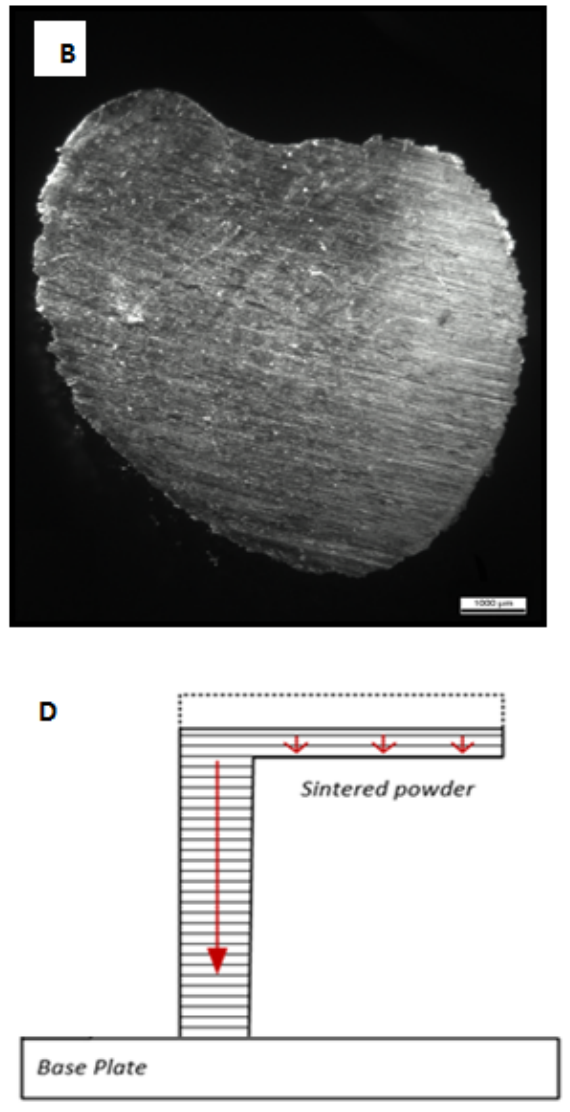

heat dissipation from the top overhanging circular features to the base, resulting in the overheating. Furthermore, the heat generated in the part could not be efficiently transferred to the sintered powder due to internal voids present between the powder particles.

From the $t$-Test analysis carried out for the scenario, it can be observed from the results in Table 4 that the $p$-values for the $X$ and $Y$ features are less than the 0.05 level of signifi- 
cance, indicating that part features vary significantly from the mean diameter. In contrast, it resulted that the com- ponent geometries fabricated along the $Z$-direction do not vary significantly with the mean population.

TABLE 4

ONE SAMPLE $T$-TEST ANALYSIS FOR SCENARIO 3

\begin{tabular}{llllll}
\hline \hline & $N$ & Mean & Std. Deviation & Std. Error Mean & $p$-Value \\
\hline Diameter $(X$-axis) & 3 & 9164.2867 & 57.46007 & 33.17459 & .001 \\
Diameter ( $Y$-axis) & 3 & 8928.8050 & 88.35350 & 51.01092 & .003 \\
Diameter $(Z$-axis) & 3 & 8012.9733 & 362.45153 & 209.26149 & .956 \\
\hline \hline
\end{tabular}

\section{Scenario 4-Overhanging Part Features Built at $60^{\circ}$ Angle}

As can be noted in Figure 6, the same pattern was observed, i.e., whilst there is minimal dimensional variation in the $Z$ direction, the cross-sections in the $X$ and $Y$ directions are characterised by high dimensional variations, mainly due to the tear-drop formation and a larger magnitude of swelling.
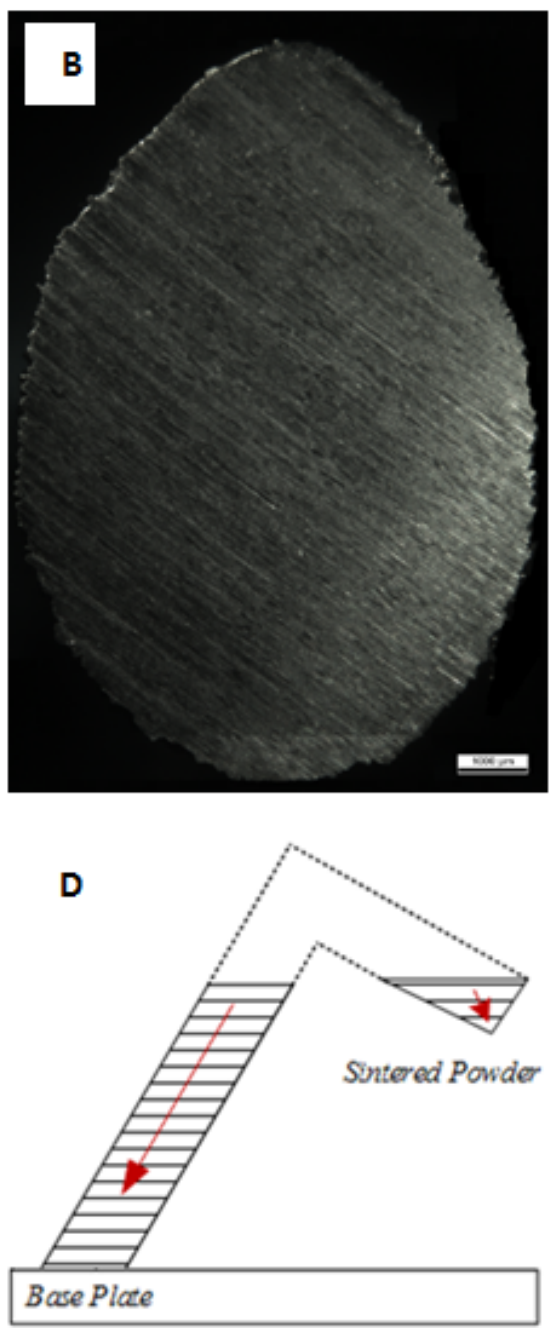

Fig. 6. Part 4 (A)-Cross-sectional view $X$ direction (B)-Cross-sectional view $Y$ direction (C)-Cross-sectional view $Z$ direction, (D)-Schematic diagram of part indicating the heat transfer direction 
As confirmed in the preceding scenario, effective heat dissipation plays a major role. As can be noted in Figure 6D, the downward-inclined cylindrical features imply that there was minimal heat transfer from the part being melted to the base plate, resulting in shrinkage and severe swelling. Also, warping of the part was clearly visible during the building of the first horizontal layers, which were built directly on sintered powder. Warping refers to excessive distortional change in a processed part, often resulting in the bending of the part, which occurs due to the rapid melting and excessive part overheating. Although the warp developed was rather small, excessive part warping can cause the part to bend upwards and collide with the rake during its movement, resulting in misalignment of the layers and possible damage to the rake.

In order to minimize the shrinkage effects as well as the excessive swelling, it is suggested to optimise the preheat process parameters and using support structures. The former is directed at reducing the internal stresses and warping by achieving more uniform temperature. However, this solution can still be insufficient if the electron beam generates very high energy, and excessive part overheating will result in shrinkage and swelling. Besides using support structure, altering the part orientation to minimise the overheating would be the optimum solution, which, however, is limited by the geometry of the model. The larger tear-drop formation observed in this scenario can be attributed to the lack of a support structure. Since support structures were not used, the cooling process was prolonged, causing shrinkage to be more severe.

The observation made with the stereomicroscope was confirmed with the One-Sample $t$-test. The analysis establishes that in the case of the $X$ and $Y$ parts oriented features, the Alternative Hypothesis applies, i.e., the sample means differ significantly from the specified value. In the case of the feature in the $Z$ orientation, the Null hypothesis applies.

TABLE 5

ONE SAMPLE $T$-TEST ANALYSIS FOR SCENARIO 3

\begin{tabular}{llllll}
\hline \hline & $N$ & Mean & Std. Deviation & Std. Error Mean & $p$-Value \\
\hline Diameter $(X$-axis) & 3 & 11233.1993 & 312.84149 & 180.61912 & .003 \\
Diameter ( $Y$-axis) & 3 & 11203.8867 & 651.97680 & 376.41898 & .014 \\
Diameter $(Z$-axis $)$ & 3 & 7898.5177 & 71.49922 & 41.28009 & .133 \\
\hline \hline
\end{tabular}

\section{E. Discussion}

From the analysis carried out, it was noted that there are close dimensional characteristics in the part features built along the $Z$-axis across all the scenarios considered. Furthermore, whilst the $X$ and $Y$ oriented features in Scenarios 1 and 2 are comparable to the mean value, the $X$ and $Y$ oriented features in Scenarios 3 and 4 differ significantly.
Figure 7 graphically summarises the dimensional variations among the part features in the $X, Y$ and $Z$ axes under the different scenarios. It can also be observed that there is minimal difference between the $X$ and $Y$ orientations across all the scenarios, mainly due to the fact that the EBM machine adopts an alternating scan method, i.e., the scanning direction alternates by $90^{\circ}$ for every layer.

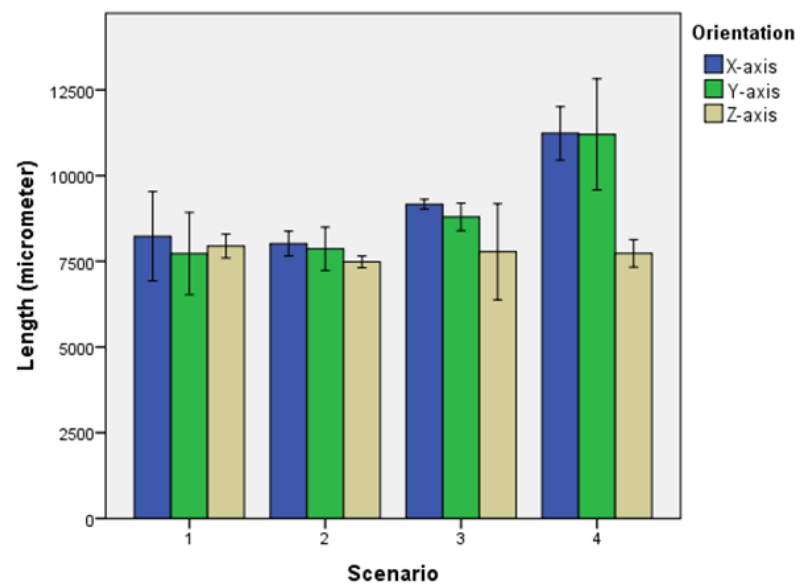

Fig. 7. Dimensional variations among the part features in the $X, Y$ and $Z$ axes under the different scenarios 
A Tukey posthoc test, presented in Table 6, was carried in order to compare the mean diameter of part features built in the $X, Y$ and $Z$ directions under different scenarios par wise. A 0.05 level of significance was adopted. In this case, in Scenarios 1 and 2, the Null Hypothesis is accepted implying that the mean diameters for part features built in different orientations vary marginally. However, for Scenarios 3 and 4, the Alternative Hypothesis is accepted, meaning that mean diameters for the features built in different scenarios vary significantly. As indicated earlier, this is mainly due to the inefficient heat transfer from the over-hanging parts to the base plate. The dimensional inaccuracy increased sharply in the part built in the fourth scenario since there was no heat flow from the overhanging features to the base plate. This resulted in high heat accumulation causing severe uniform temperature. However, this solution can still be insufficient if the electron beam generates very high energy, and excessive part overheating will result in shrinkage and swelling. Besides using support structure, altering the part orientation to minimise the overheating would be the optimum solution, which, however, is limited by the geometry of the model. The larger tear-drop formation observed in this scenario can be attributed to the lack of a support structure. Since support structures were not used, the cooling process was prolonged, causing shrinkage to be more severe.

TABLE 6

TUKEY POST-HOC TEST

\begin{tabular}{lllll}
\hline \hline Scenario & Orientation & Mean & Std. Deviation & $p$-Value \\
\hline 1 & X-axis & 8230.07 & 524.04 & 0.392 \\
& Y-axis & 7724.71 & 481.84 & \\
& Z-axis & 7944.56 & 139.74 & \\
2 & X-axis & 8015.76 & 145.66 & 0.624 \\
& Y-axis & 7864.94 & 253.69 & \\
3 & Z-axis & 7485.51 & 68.82 & \\
& X-axis & 9164.29 & 57.46 & 0.006 \\
& Y-axis & 8795.47 & 160.92 & \\
4 & Z-axis & 7779.64 & 565.08 & \\
& X-axis & 11233.20 & 312.84 & 0.000 \\
& Y-axis & 11203.89 & 651.98 & \\
& Z-axis & 7731.85 & 161.32 & \\
\hline \hline
\end{tabular}

From this study, it can be concluded that the part built in the second scenario, despite shrinkage, has the optimum dimensional accuracy. This indicates that support structures are an effective means for heat dissipation. However, an aspect that was not taken into account in the second scenario is that the removal of support structures can leave a mark on the part, affecting the dimensional accuracy. This effect can be significant, considering the small part dimensions.

\section{CONCLUSION}

This study was aimed at identifying causes of dimensional inaccuracies and defects generated in EBM parts built in four different scenarios. These scenarios reflect common defects associated with different orientation positions of the CAD parts and also defects related to overhanging parts. The common defect that emerged when the model was built directly on the base plate is incomplete part features since the first few layers of the model adhere to the base plate. When the part was built on a support structure, the com- mon defect present was shrinkage, whilst swelling was the defect that mostly occurred in overhanging part features due to the lack of effective heat transfer to the base plate. From the analysis carried out, it resulted that the use of a support structure is the most effective means to obtain high dimensional accuracy and minimise defects associated with overheating. It resulted that there were no statistically significant dimensional inaccuracies in the part features built in the $Z$ direction. Thus, whilst building part feature in the $Z$ direction is recommended in terms of dimensional quality, in case of complex medical or automotive parts with complex overhanging part features, the use of support structures is inevitable in order to obtain adequate dimensional quality. 


\section{REFERENCES}

[1] M. Cronskär, L.-E. Rännar, and M. Bäckström, "Production of customized hip stem prostheses: A comparison between machining and additive manufacturing,"' Rapid Prototyping Journal, vol. 19, no. 5, pp. 365-372, 2013. doi: https://doi. org/10.1108/rpj-07-2011-0067

[2] C. M. Tung, "Vertical integration for smart manufacturing-the dynamic capability perspective," Journal of Advances in Technology and Engineering Studies, vol. 4, no. 2, pp. 70-78, 2018. doi: https://doi.org/10.20474/jater-4.2.3

[3] K. V. Wong and A. Hernandez, "A review of additive manufacturing," International Scholarly Research Notices, vol. 2, no. 12, pp. 1-10, 2012. doi: https://doi.org/10.5402/2012/208760

[4] D. H. Abdeen and B. R. Palmer, "Effect of processing parameters of electron beam melting machine on properties of Ti-6Al-4V parts," Rapid Prototyping Journal, 2016. doi: https://doi.org/10.1108/rpj-09-2014-0105

[5] P. Drescher, M. Sarhan, and H. Seitz, "An investigation of sintering parameters on titanium powder for electron beam melting processing optimization," Materials, vol. 9, no. 12, pp. 974-984, 2016. doi: https://doi.org/10.3390/ ma9120974

[6] E. K. Law and W. K. Yung, "Manufacturing line balance and impacts on complexities," International Journal of Technology and Engineering Studies, vol. 3, no. 4, pp. 141-146, 2017. doi: https://doi.org/10.20469/ijtes.3.40002-4

[7] K. Senthilkumaran, P. M. Pandey, and P. Rao, "Influence of building strategies on the accuracy of parts in selective laser sintering," Materials \& Design, vol. 30, no. 8, pp. 2946-2954, 2009. doi: https://doi.org/10.1016/j.matdes.2009.01.009

[8] T. Grimm, G. Wiora, and G. Witt, "Characterization of typical surface effects in additive manufacturing with confocal microscopy," Surface Topography: Metrology and Properties, vol. 3, no. 1, pp. 1-14, 2015. doi: https://doi.org/10.1088/ 2051-672x/3/1/014001

[9] H. C. Cheng, S. Y. Yun, and T. H. Wen, "Automatic venipuncture insertion point recognition based on machine vision," Journal of Advances in Technology and Engineering Studies, vol. 4, no. 5, pp. 186-190, 2018. doi: https://doi.org/10. 20474/jater-4.5.1

[10] P. Alexander, S. Allen, and D. Dutta, "Part orientation and build cost determination in layered manufacturing," Computer-Aided Design, vol. 30, no. 5, pp. 343-356, 1998. doi: https://doi.org/10.1016/s0010-4485(97)00083-3

[11] H. Khalid Rafi, K. Nadimpalli, T. Starr, and B. Stucker, "Defect formation in ebm parts built in horizontal orientation," in Solid Freeform Fabrication Symposium Proceedings, New York, NY, 2012.

[12] T. Grimm, User's Guide to Rapid Prototyping. California, CA: Society of Manufacturing Engineers, 2004.

[13] M. M. Yaacob, A. R. Hussein, and M. F. Othman, "A computer program for fault diagnosis and assessment of quality insulation oil in power transformer based dissolved gas analysis," International Journal of Technology and Engineering Studies, vol. 1, no. 3, pp. 98-106, 2015. doi: https://doi.org/10.20469/ijtes.40005-3

[14] M. T. Hayasi and B. Asiabanpour, "A new adaptive slicing approach for the Fully Dense Freeform Fabrication (FDFF) process," Journal of Intelligent Manufacturing, vol. 24, no. 4, pp. 683-694, 2013. doi: https://doi.org/10.1007/ s10845-011-0615-4

[15] M. Kahnert, S. Lutzmann, and M. Zaeh, "Layer formations in electron beam sintering," in Solid Freeform Fabrication Symposium, Kulalumpur, Malaysia, 2007.

[16] E. Attar, Simulation of Selective Electron Beam Melting Processes. New York, NY: Wiley and John Sons, 2011.

[17] J. A. Helsen and Y. Missirlis, Biomaterials: A Tantalus Experience. Oxford, UK: Springer Science \& Business Media, 2010.

[18] A. Safdar, H. He, L.-Y. Wei, A. Snis, and L. E. C. de Paz, “Effect of process parameters settings and thickness on surface roughness of ebm produced ti-6al-4v," Rapid Prototyping Journal, vol. 18, no. 5, pp. 401-408, 2012. doi: https://doi. org/10.1108/13552541211250391

[19] M. Koike, P. Greer, K. Owen, G. Lilly, L. E. Murr, S. M. Gaytan, E. Martinez, and T. Okabe, "Evaluation of titanium alloys fabricated using rapid prototyping technologies electron beam melting and laser beam melting," Materials, vol. 4, no. 10, pp. 1776-1792, 2011. doi: https://doi.org/10.3390/ma4101776 